\section{Five-year incidence and progression of vascular retinopathy in persons without diabetes: the Blue Mountains Eye Study}

S Cugati, L Cikamatana, JJ Wang, A Kifley, G Liew and P Mitchell

\begin{abstract}
Purpose To assess the 5-year incidence of vascular retinopathy and its associations in an older nondiabetic population.

Methods The Blue Mountains Eye Study examined 3654 residents aged $49+$ years (82.4\% response rate) during 1992-1994, and re-examined 2335 (75.1\% of survivors) during 1997-1999. Retinopathy lesions (microaneurysms, haemorrhages, hard or soft exudates) were assessed from 6-field retinal photographs in persons without diabetes. Incident retinopathy was assessed in those at risk. Hypertensive status was defined following the WHO/International Society of Hypertension guidelines.
\end{abstract}

Results Of the 2335 re-examined, 195 had retinopathy lesions at baseline and $\mathbf{1 7 2 5}$ were at risk of retinopathy after excluding subjects with diabetes $(n=261)$, retinal vein occlusion $(n=52)$ or missing/un-gradable photographs $(n=102)$. The cumulative 5-year incidence was 9.7\% (95\% confidence intervals (CI) $8.3-11.1 \%$ ). Age was the only factor significantly associated with incident retinopathy ( $P$ for trend $=0.012$ ). Neither fasting blood glucose (age-sex-adjusted $P=0.147$ ) nor hypertension (adjusted $P$ for trend $=0.43$ ) was associated with incident retinopathy. Of the 195 with retinopathy lesions at baseline, $3.5 \%$ developed diabetes, $13.3 \%$ progressed, and 72.3\% regressed/disappeared over 5 years. Progression was positively associated with elevated blood pressure (BP) (adjusted odds ratio (OR) 1.3, 95\% CI 1.1-1.6 per $10 \mathrm{mmHg}$ systolic BP) and inversely associated with fasting glucose level (OR 0.36, CI 0.14-0.92 per $\mathrm{mmol} / \mathrm{l}$ increase). Aspirin use was weakly associated with regression (OR 2.4, CI 1.0-6.0).

Conclusions Over 5 years, retinopathy developed in $10 \%$ of older people without diabetes, while $72 \%$ of baseline lesions regressed. Age was significantly associated with the development of these lesions. Eye (2006) 20, 1239-1245. doi:10.1038/sj.eye.6702085; published online 16 September 2005

Keywords: retinopathy; incidence; non-diabetic; population-based; Blue Mountain's Eye Study

\section{Introduction}

Vascular retinopathy lesions, including microaneurysms, haemorrhages, hard and soft exudates (cotton wool spots), intraretinal microvascular abnormalities, and retinal venous beading frequently occur in persons with diabetes, where they are termed diabetic retinopathy. These lesions, however, can also occur in persons with conditions other than diabetes, ${ }^{1}$ such as hypertension, ${ }^{2-6}$ carotid atherosclerotic disease, ${ }^{7}$ blood dyscrasias, ${ }^{8}$ systemic infections, ${ }^{9-11}$ and those who have previously undergone radiotherapy. ${ }^{12}$ Recent population-based studies in older persons without diabetes have indicated a link between the presence of these retinopathy lesions and an increased risk of subsequent stroke. ${ }^{13}$ Retinopathy lesions in persons without diabetes have also been suggested to be associated with renal dysfunction, ${ }^{14}$ risk of congestive heart failure, ${ }^{15}$ or coronary heart disease in high-risk men. ${ }^{16}$
Department of

Ophthalmology, Centre for Vision Research, University of Sydney, Sydney, Australia

Correspondence: P Mitchell, Centre for Vision Research, Department of Ophthalmology, University of Sydney, Westmead Hospital, Hawkesbury Rd, Westmead, New South Wales 2145, Australia Tel: + 61298457960 ; Fax: + 61298458345 . E-mail: paul_mitchell@ wmi.usyd.edu.au

Received: 25 April 2005 Accepted in revised form: 2 August 2005 Published online: 16 September 2005

Proprietary interest: None 
In general older populations, the prevalence of vascular retinopathy in persons without diabetes varied depending on the detection methods used. In studies where the diagnosis was made during a clinical examination, ${ }^{17,18}$ reported prevalence has ranged from $0.4^{17}$ to $0.8 \% .^{18}$ In studies in which retinal photography was performed and lesions were detected via photographic grading, the prevalence has been found to be much higher: $7.8 \%$ in the Beaver Dam Eye Study (BDES), ${ }^{2} 8.3 \%$ in the Cardiovascular Health Study (CHS), ${ }^{19}$ and $9.8 \%$ in the Blue Mountains Eye Study (BMES). ${ }^{5}$ The BDES reported a 5-year retinopathy incidence of $6.0 \%$ in persons aged $43-86$ years and without diabetes ${ }^{4}$. The Hoorn Study ${ }^{20}$ reported a 9-year incidence of $7.3 \%$ in a random sample of Dutch persons aged 50-74 years with normal glucose metabolism. Aside from these two reports, there have been no other population-based incidence data on retinopathy lesions in older persons without diabetes.

We previously reported the prevalence of retinopathy in persons without diabetes from an older Australian population. ${ }^{5}$ We now report the 5-year incidence of retinopathy and associated factors from the same study population. We also report the progression over time of retinopathy lesions detected at baseline.

\section{Materials and methods}

The Blue Mountains Eye study is a population-based cohort study of an urban population aged 49 years or older at baseline; survey methods and procedures have been described previously. ${ }^{21,22}$ Of 4433 eligible residents, baseline examinations (BMES I) were performed on 3654 individuals between 1992 and 1994 (participation rate $82.4 \%$ ). The 5-year follow-up eye examinations (BMES II) were conducted between 1997 and 1999. After excluding 543 subjects who had died since BMES I, 2335 of the survivors $(75.1 \%)$ participated in BMES II. The study was approved by the Western Sydney Area Human Ethics Committee. Written, informed consent was obtained from all subjects. For the present report, subjects with either diabetes (ie, physician diagnosed diabetes and use of diabetic medication or diet control, or fasting glucose $\geq 7.0 \mathrm{mmol} / 1$ at the study examination $)^{23}$ or signs of retinal vein occlusion, either at baseline or follow-up, were excluded from the analysis.

A baseline measure of blood pressure (BP) was recorded after the subject had been seated quietly for at least $5 \mathrm{~min}$. We applied the 2003 World Health Organization (WHO)/International Society of Hypertension (ISH) guidelines to classify BP. ${ }^{24}$ Subjects were classified as prehypertensive if systolic BP (SBP) was $120-139 \mathrm{mmHg}$ or diastolic BP (DBP) $80-89 \mathrm{mmHg}$, as hypertensive stage I if SBP was $140-159 \mathrm{mmHg}$ or DBP
90-99 $\mathrm{mmHg}$ and as stage II or above if the subject was previously diagnosed as hypertensive and was using antihypertensive medications or had a SBP $\geq 160 \mathrm{mmHg}$ or DBP $\geq 100 \mathrm{mmHg}$ at examination.

Impaired fasting glucose (IFG) ${ }^{23}$ was defined when fasting blood glucose at either examination was $>6.0 \mathrm{mmol} / 1$ but $<7.0 \mathrm{mmol} / 1$. Body mass index (BMI) was calculated as weight $(\mathrm{kg}) /$ height $^{2}\left(\mathrm{~m}^{2}\right)$ and obesity was defined as BMI $\geq 30 \mathrm{~kg} / \mathrm{m}^{2}$. Hyperlipidaemia was defined as serum triglycerides $\geq 2.0 \mathrm{mmol} / 1$ and highdensity lipoprotein (HDL) cholesterol $\leq 1.0 \mathrm{mmol} / 1$. Subjects were classified as smokers if they currently smoked or had stopped smoking within the last year. Subjects were considered to be regular drinkers if they consumed $\geq 7$ alcoholic drinks per week. The following variables were used either continuously or as quartiles: $\mathrm{BP}$, triglycerides, cholesterol, HDL, fibrinogen, glucose, white cell count, and haemoglobin levels. Regular aspirin users were defined as participants taking one or more aspirin per week over the past year. Renal function was estimated based on serum creatinine and creatinine clearance (using Cockcroft Gault formula). Adjusted creatinine clearance was then calculated as creatinine clearance $\times(1.73 /$ body surface area $){ }^{25}$

We took $30^{\circ}$ stereoscopic retinal photographs (Zeiss FF3, Carl Zeiss, Oberkochen, Germany) of Diabetic Retinopathy Study fields 1 (optic disc) and 2 (macula) and nonstereoscopic photographs of fields 3 (temporal), fields 4 and 5 (upper and lower vascular arcades) and a field nasal to, but including, part of the optic disc. Fields 4 and 5 (upper and lower vascular arcades) were slightly modified to place the vascular arcades slightly closer to the centre of the photograph.,26 Photographs were graded in a masked manner for the presence of vascular retinopathy lesions, following the modified ETDRS classification of diabetic retinopathy. ${ }^{27,28}$ Retinopathy was diagnosed if any of the following lesions were present:

(1) microaneurysms, (2) blot or flame shaped haemorrhages beyond 0.5 disc diameter from the disc margin, (3) hard exudates or, (4) cotton wool spots. The level of retinopathy was further subclassified as either mild or advanced. Persons with only a single microaneurysm or a single haemorrhage present in one or both eyes were classified as mild retinopathy. Advanced retinopathy included multiple haemorrhages and/or microaneurysms, or the presence of hard exudates or cotton wool spots in either eye. Retinal photographs were digitised and all vessels passing through a circumferential zone 0.5-1.0 disc diameter from the optic disc was measured using 'Retinal Analysis' software package (Department of Ophthalmology \& Visual Science, University of Wisconsin-Madison, Madison, WI, USA). The ParrHubbard formula was used to standardise arteriolar and 
venular calibers of each eye, summarised as central retinal arteriolar equivalent and central retinal venular equivalent. $^{29}$

The progression of retinopathy was assessed in subjects with retinopathy lesions detected at baseline. Progression of retinopathy was defined if there was an increase in the number of pre-existing retinopathy lesions, or a new lesion type developed at the 5-year follow-up examination (regardless of the number of preexisting lesions). Regression was defined if the number of lesions decreased and disappearance was defined if all lesions detected at baseline were absent at follow-up.

SAS (version 8.2, SAS Institute Inc., Cary, NC, USA) was used to perform statistical analyses. Between-group differences in the proportion of participants with incident retinopathy were assessed using $\chi^{2}$ tests of independence. Trends were tested for significance using Cochran-Mantel-Haenszel $\chi^{2}$ statistic. Logistic regression was then used to assess associations with retinopathy incidence, progression, or regression after adjusting for age and sex, and variables that remained significant in the multivariable-adjusted model. Variables tested for association with retinopathy incidence, progression, or regression included baseline BP, smoking, BMI, cholesterol, triglycerides, HDL, white cell count, haemoglobin, fibrinogen, regular use of aspirin or steroids, and retinal vessel diameters. Relative risks for regression of retinopathy were assessed using the modified Poisson as described by Zou. ${ }^{30}$ With $80 \%$ power and a significance level at 0.05 , the smallest detectable risk difference in retinopathy incidence between participants with stage II hypertension and normotensive persons is 1.6 in this study.

\section{Results}

Among the 2335 participants examined after 5-year follow-up, we excluded 195 (8. 3\%) with retinopathy lesions at baseline, and $261(11.2 \%)$ persons with diabetes, $52(2.2 \%)$ with central or branch retinal vein occlusion and $102(4.4 \%)$ with no or ungradable photos at either exam, leaving 1725 subjects (73.9\%), who were considered at risk of incident retinopathy.

\section{Incident retinopathy lesions}

The cumulative 5-year incidence of any retinopathy was 9.7\%. (95\% confidence intervals (CI) $8.3-11.1 \%$ ). The incidence of mild retinopathy was $7.2 \%$ and advanced retinopathy $2.5 \%$. Of 168 participants with any retinopathy, 141 had lesions in unilateral and 27 had lesions in bilateral eyes. In the advanced retinopathy group, the number of microaneurysms and/or haemorrhages ranged between 2 and 5 while the overall number of these lesions ranged between 2 and 8

(Figure 1). The age-specific incidence of any retinopathy (either mild or advanced) was $8.2 \%$ in persons aged $<60$ years, $9.2 \%$ in persons aged $60-69$ years, $12.0 \%$ in persons aged $70-79$ years, and $15.7 \%$ among persons older than 80 years at baseline $(P$ for trend $=0.012$ ). Although the incidence of retinopathy was higher in women $(6.4 \%)$ than in men $(3.4 \%)$, this difference was not statistically significant $(P=0.055)$.

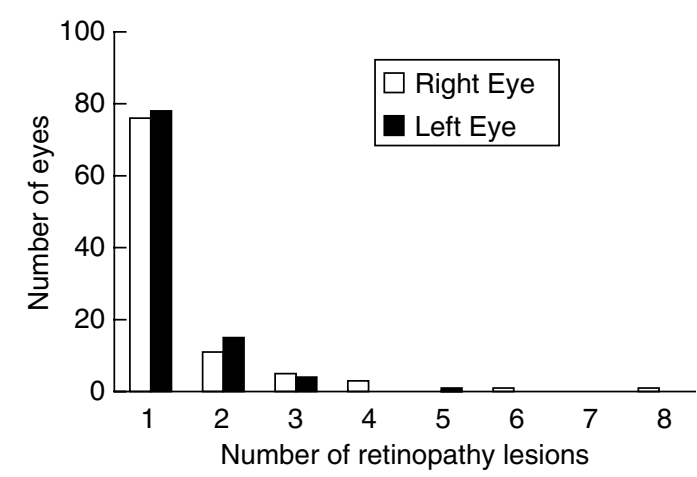

Figure 1 Number of retinopathy lesions by each eye.

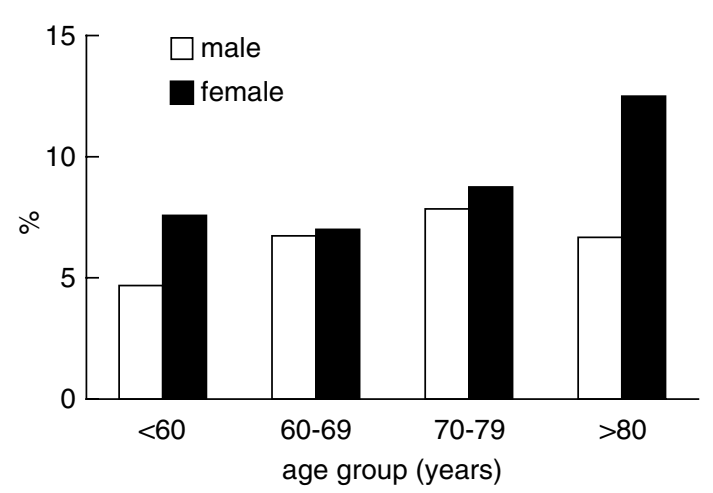

Figure 2 Age and gender-specific incidence of mild retinopathy in persons without diabetes.

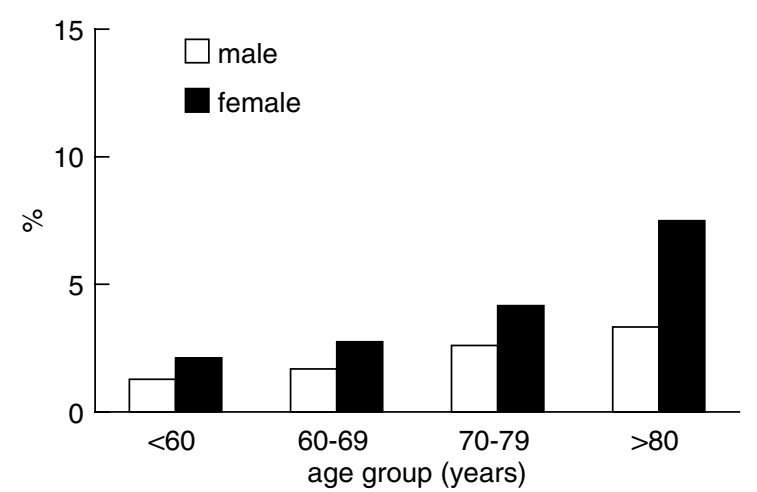

Figure 3 Age and gender-specific incidence of advanced retinopathy in persons without diabetes. 
The age- and gender-specific incidence of mild and advanced retinopathy is shown in Figures 2 and 3. Advancing age was found to be statistically significantly associated with the incidence of retinopathy, as shown in Table 1. After adjusting for age and sex, each $\mathrm{mmol} / \mathrm{l}$ increase in triglyceride level was significantly associated with a reduced risk of incident mild retinopathy (odds ratio (OR) 0.76, CI 0.59-0.97). Similarly, each mmol/1 increase in cholesterol level was associated with a reduced risk of developing any (OR 0.78 , CI 0.66-0.93) or mild retinopathy (OR 0.79, CI 0.66-0.96). These associations remained after further adjusting for the use of lipid lowering agents.
A crude association between BP and incident retinopathy was evident: increasing quartiles of SBP were associated with increased incidence of any retinopathy ( $P$ for trend $=0.026$ ) as shown in Table 2; this, however, became nonsignificant after adjusting for age and gender $(P$ for trend $=0.17$ ). Retinopathy lesions developed more frequently in persons with stage II $(11.0 \%)$ or stage I hypertension $(9.6 \%)$ compared to persons with normal BP (7.9\%) and those with prehypertension (8.2\%). The trend for these associations was not statistically significant after adjusting for age and sex $(P$ for trend $=0.43)$. Increasing SBP as a continuous measurement was also not significantly associated with

Table 1 Associations between incident retinopathy in persons without diabetes and different variables, as age-sex adjusted OR with $95 \%$ CI

\begin{tabular}{lccc}
\hline & $\begin{array}{c}\text { Any retinopathy } \\
\text { OR }(95 \% \text { CI })\end{array}$ & $\begin{array}{c}\text { Mild retinopathy } \\
\text { OR }(95 \% \text { CI })\end{array}$ & $\begin{array}{c}\text { Advanced retinopathy } \\
\text { OR }(95 \% \text { CI })\end{array}$ \\
\hline Age & $1.03(1.01-1.05)$ & $1.03(1.00-1.05)$ & $1.04(1.01-1.08)$ \\
Sex & $0.72(0.52-1.01)$ & $0.78(0.53-1.14)$ & $0.58(0.30-1.11)$ \\
Glucose $(\mathrm{mmol} / \mathrm{l})$ & $1.26(0.92-1.72)$ & $1.35(0.94-1.92)$ & $1.04(0.57-1.90)$ \\
IFG $(\mathrm{mmol} / \mathrm{l})$ & $1.51(0.66-3.43)$ & $1.46(0.57-3.79)$ & $1.63(0.38-7.04)$ \\
Hyperlipidaemia $(\mathrm{mmol} / \mathrm{l})$ & $0.7(0.33-1.48)$ & $0.56(0.22-1.4)$ & $1.21(0.36-4.09)$ \\
Triglycerides $(\mathrm{mmol} / \mathrm{l})$ & $0.87(0.71-1.06)$ & $0.76(0.59-0.97)$ & $1.14(0.86-1.50)$ \\
Cholesterol $(\mathrm{mmol} / \mathrm{l})$ & $0.78(0.66-0.93)$ & $0.79(0.66-0.96)$ & $0.78(0.58-1.06)$ \\
Use of lipid-lowering drugs & $0.69(0.27-1.74)$ & $0.75(0.27-2.1)$ & $0.51(0.07-3.78)$ \\
HDL $(\mathrm{mmol} / \mathrm{l})$ & $0.90(0.60-1.37)$ & $1.09(0.69-1.72)$ & $0.49(0.21-1.16)$ \\
Smoking & $1.16(0.7-1.92)$ & $1.17(0.66-2.07)$ & $1.13(0.43-2.95)$ \\
Alcohol $(>7$ drinks/week) & $0.80(0.55-1.15)$ & $0.77(0.51-1.18)$ & $0.87(0.44-1.72)$ \\
BMI $\left(\mathrm{kg} / \mathrm{m}^{2}\right)$ & $1.02(0.98-1.06)$ & $1.01(0.96-1.05)$ & $1.05(0.98-1.12)$ \\
Obesity & $1.41(0.93-2.14)$ & $1.30(0.80-2.12)$ & $1.73(0.84-3.58)$ \\
\hline
\end{tabular}

${ }^{a}$ Excluding retinal vein occlusions.

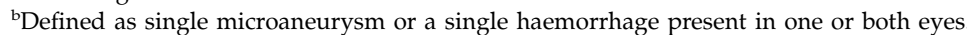

'Defined as multiple haemorrhages and/or microaneurysms, or the presence of cotton wool spots, or hard exudates in either eye.

Table 2 Association between presence of systolic blood pressure or presence of hypertension and development of retinopathy lesions in persons without diabetes

\begin{tabular}{|c|c|c|c|c|c|c|c|c|c|}
\hline & \multicolumn{3}{|c|}{ Any retinopathy } & \multicolumn{3}{|c|}{ Mild retinopathy } & \multicolumn{3}{|c|}{ Advanced retinopathy } \\
\hline & \multirow[t]{2}{*}{$\mathrm{N}(\%)$ affected } & \multicolumn{2}{|c|}{ Age-sex adjusted } & \multirow[t]{2}{*}{$\mathrm{N}(\%)$ affected } & \multicolumn{2}{|c|}{ Age-sex adjusted } & \multirow[t]{2}{*}{$\mathrm{N}(\%)$ affected } & \multicolumn{2}{|c|}{ Age-sex adjusted } \\
\hline & & $O R^{\mathrm{a}}$ & $95 \% C I^{\mathrm{a}}$ & & $O R^{\mathrm{a}}$ & $95 \% C I^{\mathrm{a}}$ & & $\mathrm{OR}^{\mathrm{a}}$ & $95 \% C I^{\mathrm{a}}$ \\
\hline Low/normal BP & $6(7.9)$ & $1.0^{\mathrm{b}}$ & & $6(7.9)$ & $1.0^{\mathrm{b}}$ & & $0(0)$ & $1.0^{\mathrm{b}}$ & \\
\hline Prehypertension & $39(8.2)$ & $1.0^{\mathrm{b}}$ & & $29(6.2)$ & $1.0^{\mathrm{b}}$ & & $10(2.2)$ & $1.0^{\mathrm{b}}$ & \\
\hline Hypertension stage 1 & $47(9.6)$ & 1.11 & $0.72-1.72$ & $34(7.2)$ & 1.05 & $0.64-1.72$ & $13(2.9)$ & 1.34 & $0.58-3.1$ \\
\hline Hypertension stage 2 & $75(11)$ & 1.18 & $0.78-1.77$ & $54(8.1)$ & 1.12 & $0.71-1.78$ & $21(3.3)$ & 1.37 & $0.62-3.02$ \\
\hline$P_{\text {(trend) }}$ & 0.102 & 0.43 & & 0.32 & 0.61 & & 0.104 & 0.46 & \\
\hline \multicolumn{10}{|l|}{ Systolic BP } \\
\hline Lowest quartile & $41(7.9)$ & 1.0 & & $32(6.3)$ & 1.0 & & $9(1.8)$ & 1.0 & \\
\hline Second quartile & $32(9.3)$ & 1.14 & $0.72-1.79$ & $22(6.6)$ & 1.09 & $0.65-1.84$ & $10(3.1)$ & 1.29 & $0.53-3.18$ \\
\hline Third quartile & $42(9.6)$ & 1.14 & $0.70-1.86$ & $31(7.3)$ & 1.02 & $0.58-1.79$ & $11(2.7)$ & 1.58 & $0.63-3.96$ \\
\hline Highest quartile & $52(12.4)$ & 1.38 & $0.88-2.17$ & $38(9.4)$ & 1.34 & $0.81-2.24$ & $14(3.7)$ & 1.53 & $0.64-3.70$ \\
\hline$P_{\text {(trend) }}$ & 0.026 & 0.17 & & 0.079 & 0.25 & & 0.14 & 0.42 & \\
\hline
\end{tabular}

${ }^{\mathrm{a}}$ OR: odds ratios; CI: $95 \%$ confidence intervals.

'In the age-sex-adjusted model, participants in the normal and prehypertension groups were categorised as the reference group. 
increased incident retinopathy (for each $10 \mathrm{mmHg}$ increase in SBP, OR 1.04, 95\% CI 0.96-1.13). Compared to persons with normal BP, those with treated but controlled hypertension were not at a higher risk of incident retinopathy (age-sex-adjusted OR $0.89,95 \% \mathrm{CI}$ $0.56-1.43)$. Nonsignificantly increased age - sex-adjusted odds for incident retinopathy were found for untreated hypertension (OR 1.24, CI 0.77-1.99) and treated but uncontrolled hypertension (OR 1.35, CI 0.83-2.21).

No significant associations were found between incident retinopathy and elevated BMI, blood glucose, hyperlipidaemia, smoking, regular drinking or smoking (Table 1), or between incident retinopathy and haemoglobin, white cell count, fibrinogen, or creatinine $(\geq 125 \mathrm{mmol} / \mathrm{l})$, or current use of aspirin or steroid medications (data not shown). There was also no association between incident retinopathy and baseline retinal vessel diameter (arteriolar narrowing, venular dilation, or arteriovenular ratio), history of stroke, acute myocardial infarction, or angina (data not shown).

\section{Progression of retinopathy detected at baseline}

Among 202 baseline participants with retinopathy lesions detected at baseline and re-examined at the 5-year follow-up, seven (3.5\%) developed diabetes during the 5year period. Of the remaining 195, baseline retinopathy lesions regressed or disappeared in 141 (72.3\%), were unchanged in $28(14.4 \%)$, and progressed in 26 persons $(13.3 \%)$. More women $(8.2 \%)$ than men $(5.1 \%)$ had retinopathy progression, but the difference was not statistically significant $(P=0.11)$.

SBP and mean arterial BP were associated with retinopathy progression. For each $10 \mathrm{mmHg}$ increase in SBP, there was a 30\% increased likelihood of retinopathy progression (OR 1.32, 95\% CI 1.07-1.63), after age-sex and blood glucose-adjustment. A similar association was observed for mean arterial BP (adjusted OR 1.47, 95\% CI 1.06-2.03). An inverse association between blood glucose level and progression of retinopathy was observed (age-sex- and mean arterial BP-adjusted OR $0.36,95 \%$ CI $0.14-0.92)$.

Aspirin intake (multivariate adjusted OR 2.4, 95\% CI 1.003-6.0) and adjusted creatinine clearance (multivariate adjusted OR 1.04, 95\% CI 1.001-1.09 for each $\mathrm{ml} / \mathrm{min} /$ $1.73 \mathrm{~m}^{2}$ ) were significantly associated with regression of retinopathy at baseline. The corresponding relative risks were 1.24 and 1.01, respectively. No other factors contributed significantly to regression of retinopathy at baseline.

\section{Discussion}

In this population-based sample of older Australians without diabetes, we found that around 10\% developed retinopathy lesions over a 5-year period. The incidence of retinopathy in our population was slightly higher than that reported by the Hoorn Study $(7.3 \% \text { over } 9 \text { years })^{20}$ and the Beaver Dam Study (6.0\% over 5 years) ${ }^{4}$ Our study population, however, was slightly older (aged 49-97 years) than the BDES population (aged 43-86 years), which could have accounted for the slightly higher incidence found. The Hoorn Study ${ }^{20}$ took two, rather than seven, retinal photographic fields, which might explain the relatively lower 9-year incidence.

Apart from age, we did not find other risk factors significantly associated with retinopathy incidence in the study population. The observed age-related increase in retinopathy incidence was consistent with our previous report ${ }^{5}$ and that from the BDES population. ${ }^{4}$ The BDES ${ }^{4}$ and the Hoorn Study ${ }^{20}$ found a slightly higher retinopathy incidence in men (6.5 and 10.5\%, respectively) than in women (5.6 and $8.6 \%$, respectively), while we observed a slightly higher retinopathy incidence in women $(6.4 \%)$ than in men $(3.4 \%)$. None of these differences were statistically significant, suggesting effects from random variation.

We previously reported a significant cross-sectional association between hypertension and retinopathy prevalence, ${ }^{5}$ similar to the $\mathrm{CHS}^{19}$ and BDES. ${ }^{2}$ In crude analyses, we observed a positive, longitudinal association between SBP and retinopathy incidence, but this became nonsignificant after adjusting for age and sex, suggesting an age-effect on the observed crude association. The failure to find a statistically significant association between BP and retinopathy incidence is likely due to insufficient study power, as the observed smallest risk difference for stage II hypertension (11.0\%) compared to normal BP (7.9\%) was $1.4(11.0 / 7.9)$ in our study population, which is smaller than the expected risk difference (1.6) that our study was able to detect.

The observed inverse associations found between baseline triglyceride level and mild incident retinopathy, and between baseline cholesterol level and incident mild or any retinopathy, are difficult to interpret. We investigated whether the use of lipid-lowering medications in subjects with increased lipid levels could have been a confounder in this association, but did not find evidence supporting this.

Known cardiovascular risk factors and blood glucose levels were not found associated with incident retinopathy in our study population. Our results are consistent with findings from the Hoorn Study. ${ }^{20}$ The Rotterdam Study, however, reported a linear association between prevalent retinopathy and random glucose levels $>6 \mathrm{mmol} / \mathrm{l}$ in their sample, which included subjects with diabetes. ${ }^{31}$

We found that only $3.5 \%$ of baseline participants without diabetes but with retinopathy at baseline 
developed diabetes over the 5-year period, including those diagnosed at the 5-year examinations. This low rate suggests that pre-clinical diabetes is unlikely to be an important cause of developing retinopathy lesions in persons without diabetes. In two-thirds of subjects with retinopathy lesions at baseline, these lesions had disappeared completely after 5 years. The observed inverse association between blood glucose level and the progression of retinopathy may have been due to chance, given the small number in this group $(n=26)$.

Strengths of our study include its representative population-based sample, use of 6-field stereoscopic retinal photographs and the detection of retinopathy lesions from masked photographic grading with confirmation by both a senior researcher (JJW) and retinal specialist (PM). Limitations include the small number of incident cases that limited our ability to detect statistical significance for some associations with small differences. There also remains the possibility of unmeasured risk factors that contributed to incident retinopathy but could not be adjusted for. The transient nature of retinopathy lesions could have led to misclassification of incident cases and controls. Survival bias could also have contributed, as persons with stage II hypertension likely to develop retinopathy may have been more likely to die before the follow-up examination.

In summary, in this older population, we found that around $10 \%$ of persons without diabetes developed incident retinopathy lesions over a 5-year period, a similar proportion to the cross-sectional prevalence of this finding. ${ }^{5}$ Apart from age and a possible effect from elevated BP, we found no other significant associations. Although the occurrence of these lesions is relatively common in older people, $72 \%$ of people with these lesions at baseline experienced regression of retinopathy over 5 years. The transient course of these lesions and their clinical significance is yet to be determined. Given the possible prognostic importance of retinopathy in relation to systemic vascular events, ${ }^{6}$ further research to elucidate causes of these signs is needed.

\section{Acknowledgements}

This study was supported by the Australian National Health \& Medical Research Council, Canberra, Australia (Grant nos. 974159, 991407, 211069, 153948).

\section{References}

1 Venkatramani J, Mitchell P. Ocular and systemic causes of retinopathy in patients without diabetes mellitus. BMJ 2004; 328: 625-629.
2 Klein R, Klein BE, Moss SE, Wang Q. Hypertension and retinopathy, arteriolar narrowing, and arteriovenous nicking in a population. Arch Ophthalmol 1994; 112: 92-98.

3 Walsh JB. Hypertensive retinopathy. Description, classification, and prognosis. Ophthalmology 1982; 89: 1127-1131.

4 Klein R, Klein BE, Moss SE. The relation of systemic hypertension to changes in the retinal vasculature: the Beaver Dam Eye Study. Trans Am Ophthalmol Soc 1997; 95: 329-348.

5 Yu T, Mitchell P, Berry G, Li W, Wang JJ. Retinopathy in older persons without diabetes and its relationship to hypertension. Arch Ophthalmol 1998; 116: 83-89.

6 Wong TY, Mitchell P. Hypertensive retinopathy. N Engl J Med 2004; 351: 2310-2317.

7 Hollenhorst RW. Ocular manifestations of insufficiency or thrombosis of the internal carotid artery. Trans Am Ophthalmol Soc 1958; 56: 474-506.

8 Reddy SC, Jackson N, Menon BS. Ocular involvement in leukemia - a study of 288 cases. Ophthalmologica 2003; 217: 441-445.

9 Vrabec TR. Posterior segment manifestations of HIV/AIDS. Surv Ophthalmol 2004; 49: 131-157.

10 Rasi V, LePore GF, Scavina P, Pisegna CL, Balacco GC. Early retinal microvascular alterations in human immunodeficiency virus seropositive asymptomatic patients. Ann Ophthalmol 1995; 27: 350-354.

11 Jabs DA, Green WR, Fox R, Polk BF, Bartlett JG. Ocular manifestations of acquired immune deficiency syndrome. Ophthalmology 1989; 96: 1092-1099.

12 Archer DB, Amoaku WM, Gardiner TA. Radiation retinopathy - clinical, histopathological, ultrastructural and experimental correlations. Eye 1991; 5: 239-251.

13 Wong TY, Klein R, Couper DJ, Cooper LS, Shahar E, Hubbard LD et al. Retinal microvascular abnormalities and incident stroke: the Atherosclerosis risk in communities study. Lancet 2001; 358: 1134-1140.

14 Wong TY, Coresh J, Klein R, Muntner P, Couper DJ, Sharrett AR et al. Retinal microvascular abnormalities and renal dysfunction: the atherosclerosis risk in communities study. J Am Soc Nephrol 2004; 15: 2469-2476.

15 Wong TY, Rosamond W, Chang PP, Couper DJ, Sharrett AR, Hubbard LD et al. Retinopathy and risk of congestive heart failure. JAMA 2005; 293: 63-69.

16 Duncan BB, Wong TY, Tyroler HA, Davis CE, Fuchs FD. Hypertensive retinopathy and incident coronary heart disease in high risk men. $\mathrm{Br}$ J Ophthalmol 2002; 86: 1002-1006.

17 Svardsudd K, Wedel H, Aurell E, Tibblin G. Hypertensive eye ground changes. Prevalence, relation to blood pressure and prognostic importance. The study of men born in 1913. Acta Med Scand 1978; 204: 159-167.

18 Leibowitz HM, Krueger DE, Maunder LR, Milton RC, Kini MM, Kahn HA et al. The Framingham Eye Study monograph: an ophthalmological and epidemiological study of cataract, glaucoma, diabetic retinopathy, macular degeneration, and visual acuity in a general population of 2631 adults, 1973-1975. Surv Ophthalmol 1980; 24: 335-610.

19 Wong TY, Hubbard LD, Klein R, Marino EK, Kronmal R, Sharrett AR et al. Retinal microvascular abnormalities and blood pressure in older people: the Cardiovascular Health Study. Br J Ophthalmol 2002; 86: 1007-1013.

20 van Leiden HA, Dekker JM, Moll AC, Nijpels G, Heine RJ, Bouter LM et al. Risk factors for incident retinopathy in a 
diabetic and nondiabetic population: the Hoorn study. Arch Ophthalmol 2003; 121: 245-251.

21 Mitchell P, Smith W, Attebo K, Wang JJ. Prevalence of agerelated maculopathy in Australia. The Blue Mountains Eye Study. Ophthalmology 1995; 102: 1450-1460.

22 Attebo K, Mitchell P, Smith W. Visual acuity and the causes of visual loss in Australia. The Blue Mountains Eye Study. Ophthalmology 1996; 103: 357-364.

23 The Expert Committee on the Diagnosis and Classification of Diabetes Mellitus. Report of the expert committee on the diagnosis and classification of diabetes mellitus. Diabetes Care 1998; 21: S5-S19.

24 Witworth JA. World Health Organization, International Society of Hypertension Writing Group. 2003 World Health Organization (WHO)/International Society of Hypertension (ISH) statement on management of hypertension. J Hypertens 2003; 21: 1983-1992.

25 Huynh SC, Kifley A, Strippoli GF, Mitchell P. Is renal impairment a predictor of the incidence of cataract or cataract surgery? Findings from a population-based study. Ophthalmology 2005; 112: 293-300.
26 Mitchell P, Smith W, Wang JJ, Attebo K. Prevalence of diabetic retinopathy in an older community. The Blue Mountains Eye Study. Ophthalmology 1998; 105: 406-411.

27 Early Treatment Diabetic Retinopathy Study Research Group. Early photocoagulation for diabetic retinopathy (Report No. 9). Ophthalmology 1991; 98: 766-785.

28 Diabetic Retinopathy Study Research Group. DRS Report Number 7: a modification of the Airlie House classification of diabetic retinopathy. Invest Ophthalmol Vis Sci 1981; 21: 210-226.

29 Leung H, Wang JJ, Rochtchina E, Tan AG, Wong TY, Hubbard LD et al. Computer-assisted retinal vessel measurement in an older population: Correlation between right and left eyes. Clin Exp Ophthalmol 2003; 31: 326-330.

30 Zou G. A modified poisson regression approach to prospective studies with binary data. Am J Epidemiol 2004; 159: 702-706.

31 Stolk RP, Vingerling JR, de Jong PT, Dielemans I, Hofman A, Lamberts SW et al. Retinopathy, glucose, and insulin in an elderly population. The Rotterdam Study. Diabetes 1995; 44: 11-15. 